\title{
Psychometric Validation of the Malaysian Chinese Version of the EORTC QLQ-C30 in Colorectal Cancer Patients
}

\author{
Bello Arkilla Magaji ${ }^{1,2,3 *}$, Foong Ming Moy ${ }^{1}$, April Camilla Roslani ${ }^{2,3}$, Chee Wei \\ Law $^{2}$, Ismail Sagap 5
}

\begin{abstract}
Background and Aims: Colorectal cancer is the second most frequent cancer in Malaysia. We aimed to assess the validity and reliability of the Malaysian Chinese version of European Organization for Research and Treatment of Cancer (EORTC) Quality of Life Questionnaire core (QLQ-C30) in patients with colorectal cancer. Materials and Methods: Translated versions of the QLQ-C30 were obtained from the EORTC. A cross sectional study design was used to obtain data from patients receiving treatment at two teaching hospitals in Kuala Lumpur, Malaysia. The Malaysian Chinese version of QLQ-C30 was self-administered in 96 patients while the Karnofsky Performance Scales (KPS) was generated by attending surgeons. Statistical analysis included reliability, convergent, discriminate validity, and known-groups comparisons. Statistical significance was based on $p$ value $\leq \mathbf{0 . 0 5}$. Results: The internal consistencies of the Malaysian Chinese version were acceptable [Cronbach's alpha $(\alpha \geq 0.70)$ ] in the global health status/overall quality of life (GHS/QOL), functioning scales except cognitive scale $(\alpha \leq 0.32)$ in all levels of analysis, and social/family functioning scale $(\alpha=0.63)$ in patients without a stoma. All questionnaire items fulfilled the criteria for convergent and discriminant validity except question number 5 , with correlation with role $(r=0.62)$ and social/family $(r=0.41)$ functioning higher than with physical functioning scales $(r=0.34)$. The test-retest coefficients in the GHS/QOL, functioning scales and in most of the symptoms scales were moderate to high $(r=0.58$ to 1.00$)$. Patients with a stoma reported statistically significant lower physical functioning $(p=0.015)$, social/family functioning $(p=0.013)$, and higher constipation $(p=0.010)$ and financial difficulty $(\mathrm{p}=\mathbf{0 . 0 3 7})$ compared to patients without stoma. There was no significant difference between patients with high and low KPS scores. Conclusions:Malaysian Chinese version of the QLQ-C30 is a valid and reliable measure of HRQOL in patients with colorectal cancer.
\end{abstract}

Keywords: EORTC QLQ-C30 - colorectal carcinoma - Malaysia Chinese - validation

Asian Pac J Cancer Prev, 16 (18), 8107-8112

\section{Introduction}

Colorectal cancer is an important public health burden throughout the world (Haggar and Boushey, 2009). Numerous researchers indicate that patients with colorectal cancer disease experience impairment in their Health-related Quality of Life (HRQOL) (Gujral et al., 2008; Kilic et al., 2012; Abu-Helalah et al., 2014). The HRQOL research in patients with cancer underwent several developments over the last three decades with European Organization for Research and Treatment of Cancer (EORTC) playing a major role (Velikova et al., 2012). For example, the development and subsequent update of the EORTC cancer core Quality of Life questionnaire (QLQ-C30) contributes significantly in this regard (King, 1996).

This EORTC QLQ-C30 popularly referred to as "core questionnaire" is applicable in all cancer patients. The questionnaire was first released in 1993. Since then it has undergone several revisions and further developments (Osoba et al., 1997). This questionnaire is frequently used in cancer of the colorectum (Hung et al., 2013; Smith et al., 2013). The questionnaire has been validated across many cultures and disease conditions and was found to have adequate acceptable psychometric properties (Schwenk et al., 2004; Huang et al., 2007; Jayasekara et al., 2008; Luckett et al., 2011; Uwer et al., 2011; Yakar and Pinar, 2013). Details of its application, interpretation and reference values for comparison among different groups of patients are readily available (King, 1996; Michelson et al., 2000; Fayers, 2001; Arraras Urdaniz et al., 2008; Finck et al., 2012). However, to the best knowledge of the researchers, the QLQ-C30 was only validated for use in Bahasa Malaysia for use in patients with breast cancer (Yusoff et al., 2010), and use to examined quality of life of colorectal cancer in Malaysia (Natrah et al., 2012; Sharifa

${ }^{1}$ Julius Centre University of Malaya, Department of Social \& Preventive Medicine, ${ }^{2}$ Department of Surgery, Faculty of Medicine, ${ }^{3}$ University of Malaya Cancer Research Institute, University of Malaya, ${ }^{5}$ Department of Surgery, Universiti Kebangsaan Malaysia Medical Centre, Kuala Lumpur, Malaysia, ${ }^{4}$ Department of Community Health, College of Health Sciences, Usmanu Danfodiyo University, Sokoto, Nigeria*For correspondence:drmagaji@siswa.um.edu.my,mbarkilla@gmail.com 
Bello Arkilla Magaji et al

Ezat et al., 2014). However, the psychometric properties of QLQ-C30 has never been examined for Malaysian Chinese language in colorectal cancer patients, despite the fact that colorectal cancer is more common among Chinese compared to other ethnic groups in Malaysia (Magaji et al., 2014; Shah et al., 2014). Thus, evaluating its psychometric properties in Malaysian Chinese patients with colorectal cancer is both necessary and timely. Therefore, in this study we aimed to evaluate the reliability and validity of the Malaysian Chinese version of the QLQ-C30 (version 3.0) in colorectal cancer patients receiving treatment at two university teaching hospitals in Kuala Lumpur, Malaysia.

\section{Materials and Methods}

This study involves colorectal cancer patients receiving treatment at the University Malaya Medical Centre (UMMC) and University Kebangsaan Malaysia medical centre (UKMMC). This study receives ethics approval from the UMMC (PPUM/UPP/300/02/02, MEC 770.2) and the UKMMC (Project code: FF-2742011). Patient's recruitment took place between February 2012 and June 2012. Demographic and clinical data was retrieved from patients' medical records using a pre-design form. The Malaysian Chinese version of the QLQ-C30 was obtained from the Quality of Life department of EORTC and was used to obtained relevant data. All participants provide written informed consent. Participants were requested to complete the questionnaire by themselves while waiting to see the doctor at the outpatient surgical clinic or immediately after the consultation. In addition, surgeons rated the patient's overall wellbeing using the Karnofsky Performance Scale (KPS). For the test-retest analysis, thirty patients were requested to complete and return another set of the questionnaire 1 to 2 weeks after the first assessment.

Descriptive analysis was performed; continuous data was summarized using mean and standard deviations while categorical data was summarized using proportions. The internal consistency of the multi-item scales was examined by the use of Cronbach's alpha coefficient. A coefficient of $\geq 0.70$ is considered acceptable. Testretest correlation coefficients were examined to test the reproducibility of the questionnaire. Multi-trait scaling analyses were used to examine the scale structures in terms of convergent and discriminant validity. A criterion considered is that each item own scale correlation should exceed 0.4 for convergent validity to be achieved. The discriminant validity measures item correlation with other scales. It is hypothesized that the item's own correlation should be higher than with the other scales. Clinically distinct group's validity was examined by comparing the scores of patients with and without stoma as well as two dichotomize KPS score of $<80 \%$, and $>80 \%$. All analyses were done via SPSS version 21.0 for Windows, (SPSS Inc., and Chicago, Illinois, USA). A two-tailed probability value of 0.05 was used to determine the level of significance. Protocol for this study was published in the BMC cancer (Magaji et al., 2012).

\section{Results}

\section{Patients characteristics}

A total of 96 patients (UMMC 50, UKMMC 46) completed the questionnaire. The mean age and standard deviation (SD) was 64 years (SD 10), range 43-83 years. Majority $(60 \%)$ were male, retired civil servants (58\%) and $21 \%$ without formal education. Stage information was missing for 9 patients. Advanced stage (Dukes' C/D) cancers account for $59 \%$. The mean KPS was $80 \%$ (SD 10 ) and $30 \%$ of patients had a stoma (Table 1 ).

Internal consistency and test-re-test correlation coefficients

The internal consistencies of the Malaysian Chinese version were acceptable with Cronbach's alpha $(\alpha \geq 0.70)$ in the global health status/overall quality of life (GHS/ QOL), and most of the functioning and multi-item symptom scales. However, the cognitive functioning scale indicated low Cronbach's alpha values across all levels of analysis (Cronbach's alpha ranges from 0.12 to 0.32). Similarly, Cronbach's alpha for the social/family functioning scale was low $(\alpha=0.63)$ in the subgroup of patients without a stoma bag (Table 2).

The test-retest coefficients in the GHS/QOL,

Table 1. Demographic and Clinical Features of the patients $(n=96)$

\begin{tabular}{lc}
\hline Characteristics & Number $(\%)$ \\
\hline Age (years) & \\
Mean (SD) & $64(10)$ \\
Range & $43-83$ \\
Gender & \\
Male & $58(60)$ \\
Female & $38(40)$ \\
Educational status & \\
Primary & $28(29)$ \\
Secondary & $19(20)$ \\
Tertiary & $3(3)$ \\
Not formally educated & $20(21)$ \\
Unknown & $26(27)$ \\
Employment status & \\
Full time & $26(27)$ \\
Retired & $56(58)$ \\
Unemployed & $7(7)$ \\
Unknown & $7(7)$ \\
Site & \\
Colon & $48(50)$ \\
Rectal & $34(36)$ \\
Unknown & $14(14)$ \\
Dukes' stage & \\
A & $4(4)$ \\
B & $26(27)$ \\
C & $16(17)$ \\
D & $41(43)$ \\
Unknown & $9(9)$ \\
Stoma & \\
Yes & $29(30)$ \\
No & $67(70)$ \\
Marnofsky performance status & \\
Range & $80(10)$ \\
\80 & $50-90$ \\
$\geq 81$ & $57(59)$ \\
\hline
\end{tabular}


Psychometric Validation of the Malaysian Chinese Version of the EORTC QLQ-C30 in Colorectal Cancer Patients

Table 2. Internal Consistency Coefficient (Malaysian Chinese)

\begin{tabular}{llll}
\hline Scale & \multicolumn{3}{c}{ Cronbach's alpha } \\
\cline { 2 - 4 } & $\begin{array}{c}\text { Overall } \\
\text { Sith } \\
\text { stoma }\end{array}$ & $\begin{array}{c}\text { Without } \\
\text { stoma }\end{array}$ \\
\hline Global health status/quality of life & & & \\
$\quad$ Global health status/quality of life & 0.91 & 0.92 & 0.89 \\
Functioning scales & & & \\
$\quad$ Physical & 0.74 & 0.73 & 0.72 \\
$\quad$ Role & 0.92 & 0.98 & 0.85 \\
Emotional & 0.79 & 0.82 & 0.78 \\
Cognitive & $0.26^{*}$ & $0.12^{*}$ & $0.32^{*}$ \\
$\quad$ Social and Family & 0.71 & 0.75 & $0.63 *$ \\
Multi-item symptoms scales & & & \\
$\quad$ Fatigue & 0.79 & 0.85 & 0.75 \\
$\quad$ Nausea/Vomiting & 0.82 & 0.85 & 0.81 \\
Pain & 0.77 & 0.81 & 0.76 \\
\hline *scale with Cronbach's alpha less than 0.65 & & &
\end{tabular}

*scale with Cronbach's alpha less than 0.65 functioning scales and in most of the symptoms scales were high ( $\mathrm{r}=0.83$ to 1.00$)$. The lowest test-retest reliability coefficients were found in appetite loss $(\mathrm{r}=0.58)$.

\section{Multi-trait scaling analyses}

All questionnaire items fulfilled the criteria for convergent and discriminant validity except question number 5 (Q5). The correlation of question number 5 with its own proposed scale (the physical functioning scale) was $r=0.34$ which was less than the minimum required coefficient of $r \geq 0.40$. On the other hand, this item's correlation with other scales such as role $(r=0.62)$ and social/family functioning ( $\mathrm{r}=0.41$ ) was higher than its correlation with its own domain (Table 3 ).

\section{Clinically distinct group comparisons}

Patients with stoma reported statistically significant lower physical functioning $(\mathrm{p}=0.015)$, social/family functioning $(\mathrm{p}=0.013)$, and higher constipation $(\mathrm{p}=0.010)$

Table 3. Multi-trait scaling analysis (Malaysian Chinese)

\begin{tabular}{|c|c|c|c|c|c|c|c|c|c|}
\hline \multirow[t]{2}{*}{ Questionnaire item } & \multicolumn{6}{|c|}{ Functioning } & \multicolumn{3}{|c|}{ Multi-item symptom scales } \\
\hline & $\begin{array}{l}\text { GHS } \\
\text { /QOL }\end{array}$ & Physical & Role & Cognitive & Emotional & $\begin{array}{l}\text { Social } \\
\text { Family }\end{array}$ & Fatigue & $\begin{array}{c}\text { Nausea } \\
\text { Vomiting }\end{array}$ & Pain \\
\hline \multicolumn{10}{|l|}{ GHS/QOL } \\
\hline 29 Global Health Status & 0.95 & 0.36 & 0.38 & 0.26 & 0.49 & 0.42 & -0.50 & -0.35 & -0.49 \\
\hline 30 Overall Quality of Life & 0.96 & 0.39 & 0.41 & 0.35 & 0.49 & 0.42 & -0.52 & -0.25 & -0.39 \\
\hline \multicolumn{10}{|l|}{ Functioning } \\
\hline \multicolumn{10}{|l|}{ Physical } \\
\hline 1 Strenuous activity & -0.27 & -0.74 & -0.41 & -0.18 & -0.11 & -0.33 & 0.41 & 0.17 & 0.29 \\
\hline 2 Long walk & -0.33 & -0.85 & -0.38 & -0.13 & -0.09 & -0.21 & 0.36 & 0.15 & 0.26 \\
\hline 3 Short walk & -0.34 & -0.78 & -0.54 & -0.13 & -0.17 & -0.32 & 0.39 & 0.32 & 0.35 \\
\hline 4 Stay in bed/chair & -0.28 & -0.72 & -0.46 & -0.18 & -0.16 & -0.25 & 0.5 & 0.19 & 0.33 \\
\hline $\begin{array}{l}5 \text { Need help in eating/ } \\
\text { dressing/washing }\end{array}$ & -0.16 & $-0.33 *$ & $-0.62 *$ & -0.18 & -0.27 & $-0.41 *$ & 0.23 & 0.06 & 0.06 \\
\hline \multicolumn{10}{|l|}{ Role } \\
\hline 6 Limited work & -0.40 & -0.62 & -0.96 & -0.30 & -0.37 & -0.52 & 0.55 & 0.23 & 0.44 \\
\hline 7 Limited hobbies & -0.40 & -0.63 & -0.96 & -0.29 & -0.35 & -0.50 & 0.51 & 0.21 & 0.37 \\
\hline \multicolumn{10}{|l|}{ Cognitive } \\
\hline 20 Concentration & -0.34 & -0.20 & -0.37 & -0.72 & -0.41 & -0.40 & 0.44 & 0.39 & 0.35 \\
\hline 25 Memory & -0.16 & -0.14 & -0.11 & -0.79 & -0.27 & -0.24 & 0.22 & -0.01 & 0.07 \\
\hline \multicolumn{10}{|l|}{ Emotional } \\
\hline 21 Tense & -0.32 & -0.12 & -0.28 & -0.33 & -0.76 & -0.36 & 0.33 & 0.27 & 0.15 \\
\hline 22 Worried & -0.35 & -0.07 & -0.25 & -0.30 & -0.84 & -0.41 & 0.37 & 0.22 & 0.20 \\
\hline 23 Irritable & -0.35 & -0.14 & -0.20 & -0.34 & -0.64 & -0.34 & 0.28 & 0.18 & 0.01 \\
\hline 24 Depressed & -0.58 & -0.32 & -0.44 & -0.43 & -0.88 & -0.61 & 0.65 & 0.33 & 0.40 \\
\hline \multicolumn{10}{|l|}{ Social/Family } \\
\hline 26 Family life & -0.41 & -0.23 & -0.28 & -0.29 & -0.49 & -0.86 & 0.53 & 0.23 & 0.41 \\
\hline 27 Social life & -0.37 & -0.47 & -0.63 & -0.43 & -0.48 & -0.90 & 0.57 & 0.34 & 0.38 \\
\hline \multicolumn{10}{|l|}{ Multi-item symptoms scales } \\
\hline \multicolumn{10}{|l|}{ Fatigue } \\
\hline 10 Need rest & -0.48 & -0.52 & -0.45 & -0.29 & -0.33 & -0.49 & 0.83 & 0.31 & 0.55 \\
\hline 12 Feel weak & -0.49 & -0.5 & -0.53 & -0.36 & -0.54 & -0.55 & 0.85 & 0.29 & 0.48 \\
\hline 18 Tired & -0.36 & -0.36 & -0.40 & -0.42 & -0.45 & -0.54 & 0.85 & 0.30 & 0.53 \\
\hline \multicolumn{10}{|l|}{ Nausea/Vomiting } \\
\hline 14 Nausea & -0.31 & -0.22 & -0.18 & -0.19 & -0.32 & -0.36 & 0.37 & 0.92 & 0.45 \\
\hline 15 Vomiting & -0.27 & -0.25 & -0.25 & -0.23 & -0.26 & -0.24 & 0.29 & 0.91 & 0.425 \\
\hline \multicolumn{10}{|l|}{ Pain } \\
\hline 9 Pain & -0.40 & -0.36 & -0.35 & -0.21 & -0.18 & -0.38 & 0.57 & 0.44 & 0.90 \\
\hline $\begin{array}{l}19 \text { Relation of pain } \\
\text { with daily activity }\end{array}$ & -0.43 & -0.32 & -0.42 & -0.26 & -0.26 & -0.43 & 0.55 & 0.41 & 0.91 \\
\hline
\end{tabular}

*GHS/QOL, Global Health Status/Quality of Life; *Question number 5 correlations with physical, role and social/family functioning scales 
Table 4. Group comparison between patients with and without a stoma

\begin{tabular}{|c|c|c|c|}
\hline \multirow[t]{2}{*}{ Domains } & \multicolumn{2}{|c|}{ Malaysian Chinese } & \multirow[b]{2}{*}{$\mathrm{p}$ - value } \\
\hline & $\begin{array}{c}\text { Stoma } \\
(\mathrm{n}=29) \\
\text { Mean (SD) }\end{array}$ & $\begin{array}{c}\text { No Stoma } \\
(\mathrm{n}=67) \\
\text { Mean (SD) }\end{array}$ & \\
\hline \multicolumn{4}{|l|}{ GHS/QOL } \\
\hline $\begin{array}{l}\text { GHS/QOL } \\
\text { Functioning scales }\end{array}$ & $62.93(28.05)$ & $69.53(20.59)$ & 0.405 \\
\hline Physical & 73.33(19.92) & $82.69(16.65)$ & $0.015^{*}$ \\
\hline Role & $74.71(34.97)$ & $87.31(21.92)$ & 0.248 \\
\hline Emotional & 79.59(19.36) & 83.95(16.81) & 0.254 \\
\hline Cognitive & $80.45(14.81)$ & $79.85(17.29)$ & 0.926 \\
\hline Social/Family & $71.26(24.76)$ & $84.32(18.77)$ & $0.013 *$ \\
\hline \multicolumn{4}{|c|}{ Multi-items symptom scales } \\
\hline Fatigue & $31.42(25.89)$ & 28.52(19.17) & 0.903 \\
\hline Nausea/Vomiting & $9.19(15.16)$ & $6.22(14.16)$ & 0.303 \\
\hline Pain & $19.54(22.30)$ & 14.68(18.92) & 0.344 \\
\hline \multicolumn{4}{|c|}{ Single item/symptoms } \\
\hline Dyspnoea & $6.89(13.74)$ & 9.95(18.36) & 0.558 \\
\hline Sleep loss & $21.84(28.56)$ & $22.39(24.19)$ & 0.697 \\
\hline Appetite loss & $10.34(20.12)$ & $10.45(19.42)$ & 0.924 \\
\hline Constipation $* *$ & $9.19(23.39)$ & $19.40(24.72)$ & $0.010 *$ \\
\hline Diarrhoea & $16.09(24.59)$ & $15.92(23.57)$ & 0.956 \\
\hline Financial difficulty & $40.23(30.05)$ & $27.86(30.48)$ & $0.037 *$ \\
\hline
\end{tabular}

GHS/QOL: Global Health Status/Quality of Life, *Significant difference $(\mathrm{p}<0.05)$

and financial difficulty ( $\mathrm{p}=0.037)$ compared to patients without a stoma (Table 4). On the other hand, there is a trend whereby patients with high mean scores for KPS reported better GHS/QOL, higher functioning and fewer symptoms. However, the differences were not statistically significant across all levels.

\section{Discussion}

The internal consistency coefficients of the Malaysian Chinese version were greater than the acceptable level of 0.70 in most of the scales examined. Three scales namely cognitive and social/family functioning scales showed low internal consistency ( $\alpha \leq 0.70)$. The cognitive functioning scale showed poor internal consistency at all levels of analysis. Our findings were similar to several other validation studies involving the EORTC QLQ-C30 in many Asian countries and language settings such as China (Cheng et al., 2011), Indonesia (Perwitasari et al., 2011), Singapore (Luo et al., 2005), Korea (Yun et al., 2004), Turkey (Guzelant et al., 2004), Iran (Hajebrahimi et al., 2013), and Malaysia (breast cancer) (Yusoff $\mathrm{N}$ et al., 2010). All these studies reported a poor internal consistency coefficient for the cognitive functioning scale (Cronbach's alpha ranges from 0.19 to 0.67 ). The cognitive functioning scale was comprised of items for memory and concentration. These two items were not necessarily strongly associated with each other (Luo et al., 2005). Therefore, a lack of high internal consistency between these two items in the scale could be expected. Thus, correlation of cognitive functioning scale with relevant clinical findings should be considered wherever necessary. While the social/family functioning scale showed poor internal consistency in patients completing the Malaysian
Chinese version, specifically patients without a stoma. Our finding is similar to the previous study by Paiva et al., 2014. This is, however, contrary to other study in which the social/family functioning scale showed high internal consistency (Perwitasari et al., 2011).

Overall, our findings indicated that each scale (except the cognitive functioning and pain scale) measured unique domains of HRQOL and, therefore, the scales can be used for group comparisons involving colorectal cancer patients. The differences observed in pain and social/ family functioning scale for patients with and without a stoma indicated that items examining some aspect of HRQOL among the two mutually exclusive groups of patients were not necessarily the same.

Moderate to high agreement was observed $(r \geq 0.50)$ in most of the scales. Our finding is similar to a previous study on a simplified Chinese version of the QLQ-C30 where a low test-retest correlation was observed in the appetite loss scale (Wan et al., 2008). Similarly, validation of the Bahasa Malaysia version of EORTC QLQ C30 among breast cancer patients also revealed low test-retest scores for the appetite loss and financial difficulty scales (Yusoff et al., 2010).

With reference to the multi-trait scaling analyses, the Malaysian Chinese versions of the QLQ-C30 met the criteria for convergent and discriminant validity with the exception of question number 5 "need help in eating/ dressing/washing". In our study, the question number 5 correlated highly with the role, cognitive, as well social/ family functioning scales compared to its intended physical functioning scale. This finding was expected since this questionnaire item had remained the most unstable item in the EORTC QLQ-C30. Numerous validation studies reported a lack of convergent and discriminant validity for 
this item (Hoopman et al., 2006; Perwitasari et al., 2011).

For example, validation of the Indonesian version of the EORTC QLQ-C30 by Perwitasari et al. (2011) showed question number 5 correlated higher with the emotional functioning scale than its proposed physical function scale.

The ability of the scales/items in the Malaysian Chinese version of the EORTC QLQ-C30 to differentiate between two clinically distinct patients groups was examined based on the presence or absence of stoma, and by two dichotomized scales of the Karnofsky Performance Scores. Patients with a stoma experienced more impairment in their physical and social/family functioning, less constipation and more financial difficulty, the lack of a stoma bag being associated with constipation. This is similar to previous studies indicating that patients with a stoma experienced negative consequences due to the stoma in their day to day functional wellbeing (Zaj $\mathrm{AC}$ et al., 2008). However, a recent Cochrane review involving thirty-five observational studies representing 5127 patients concluded that even though differences were observed between patients with and without a stoma, such differences were not consistent (Pachler and WilleJorgensen, 2012).

In the comparison between patients with low and high KPS scores $(\leq 80 \%$ and $\geq 81 \%$ ), we found that patients with a low score $(\leq 80 \%)$, experienced more dyspnoea and appetite loss compared to patients with higher KPS score $(\geq 81 \%)$ among patients completing the Malaysian Chinese version. This indicated that, in our setting, a few of the EORTC QLQ-C30 scales differentiated between patients on the dichotomized performance scale. This is comparable to studies in Indonesia (Perwitasari et al., 2011) and Singapore (Luo et al., 2005). However, the Korean version of the EORTC QLQ-C30 reported a significant difference between patients in subgroups of performance scale as measured using the Eastern Oncology Group (ECOG) scale. Patients with poor ECOG performance scores had poor GHS/QOL, functional status and more symptoms compared to those with higher ECOG scores (Yun et al., 2004).

In conclusion, our study findings are the first of its kind and indicated that the Malaysian Chinese version of the QLQ-C30 is a valid and reliable measure of HRQOL in patients with colorectal cancer. Potential uses of these questionnaires are both in clinical settings and researches. Further researches are needed to examine the potentials use of these instruments in routine clinical practice

\section{Acknowledgements}

This study was fully funded by the University of Malaya postgraduate fund with the following reference numbers (UM-IPPP PS212/2010A and PV015-2011B) and partially supported by the STeMM Programme, the University of Malaya/Ministry of Higher Education (UM/ MOHE) High Impact Research Grant (No: E00001020001).

\section{References}

Abu-Helalah MA, Alshraideh HA, Al-Hanaqta MM, et al (2014).
Quality of life and psychological well-being of colorectal cancer survivors in Jordan. Asian Pac J Cancer Prev, 15, 7653-64.

Arraras Urdaniz JI, Villafranca Iturre E, Arias de la Vega F, et al (2008). The eortc quality of life questionnaire QLQ-C30 (version 3.0). Validation study for Spanish prostate cancer patients. Arch Esp Urol, 61, 949-54.

Cheng JX, Liu BL, Zhang X, et al (2011). The validation of the standard chinese version of the european organization for research and treatment of cancer quality of life core questionnaire 30 (EORTC QLQ-C30) in pre-operative patients with brain tumor in China. BMC Med Res Methodol, 11, 56.

Fayers PM (2001). Interpreting quality of life data: populationbased reference data for the EORTC QLQ-C30. Eur $J$ Cancer, 37, 1331-4.

Finck C, Barradas S, Singer S, et al (2012). Health-related quality of life in Colombia: reference values of the EORTC QLQ-C30.Eur J Cancer Care, 21, 829-36.

Gujral S, Avery KNL, Blazeby JM (2008). Quality of life after surgery for colorectal cancer: clinical implications of results from randomised trials. Supportive Care in Cancer, 16, 127-32.

Guzelant A, Goksel T, Ozkok S, et al (2004). The european organization for research and treatment of cancer QLQ-C30: an examination into the cultural validity and reliability of the Turkish version of the EORTC QLQ-C30. Eur J Cancer Care, 13, 135-44.

Haggar FA, Boushey RP (2009). Colorectal cancer epidemiology: incidence, mortality, survival, and risk factors. Clin Colon Rectal Surg, 22, 191-7.

Hajebrahimi S, Sadeghi H, Sari RM (2013). C30 validity and reliability ICIQ-OAB questionnaire in the persian version. European Urology Supplements, 1138, 30.

Hoopman R, Muller MJ, Terwee CB, et al (2006). Translation and validation of the EORTC QLQ-C30 for use among turkish and moroccan ethnic minority cancer patients in the netherlands. European J Cancer, 42, 1839-47.

Huang CC, Lien HH, Sung YC, et al (2007). Quality of life of patients with gastric cancer in Taiwan: validation and clinical application of the Taiwan Chinese version of the EORTC QLQ-C30 and EORTC QLQ-STO22.Psychooncol, 16, 945-9.

Hung HC, Chien TW, Tsay SL, et al (2013). Patient and clinical variables account for changes in health- related quality of life and symptom burden as treatment outcomes in colorectal cancer: a longitudinal study. Asian Pac J Cancer Prev, 14, 1905-9.

Jayasekara H, Rajapaksa LC, Aaronson NK (2008). Quality of life in cancer patients in South Asia: psychometric properties of the Sinhala version of the EORTC QLQ-C30 in cancer patients with heterogeneous diagnoses. Qual Life Res, 17, 783-91.

Kilic D, Yalman D, Aksu G, et al (2012). Impact of adjuvant chemoradiotherapy for rectal cancer on the long-term quality of life and late side effects: a multicentric clinical evaluation by the Turkish oncology group. Asian Pac J Cancer Prev, 13, 5741-6.

King MT (1996). The interpretation of scores from the EORTC quality of life questionnaire QLQ-C30. Qual Life Res, 5, 555-67.

Luckett T, King MT, Butow PN, et al (2011). Choosing between the EORTC QLQ-C30 and FACT-G for measuring healthrelated quality of life in cancer clinical research: issues, evidence and recommendations. Ann Oncol, 22, 2179-90.

Luo N, Fones CS, Lim SE, et al (2005). The european organization for research and treatment of cancer quality 
Bello Arkilla Magaji et al

of Life Questionnaire (EORTC QLQ-c30): validation of English version in Singapore. Qual Life Res, 14, 1181-6.

Magaji BA, Law CW, Roslani AC, et al (2012). Health-related quality of life among colorectal cancer patients in Malaysia: A study protocol. BMC Cancer, $\mathbf{3 8 4}$.

Magaji BA, Moy FM, Roslani AC, et al (2014). Descriptive epidemiology of colorectal cancer in University Malaya medical centre, 2001 to 2010. Asian Pac J Cancer Prev, 15, 6059-64.

Michelson H, Bolund C, Nilsson B, et al (2000). Health-related quality of life measured by the EORTC QLQ-C30--reference values from a large sample of Swedish population. Acta Oncol, 39, 477-84.

Natrah MS, Ezat S, Syed MA, et al (2012). Quality of life in Malaysian colorectal cancer patients: a preliminary result. Asian Pac J Cancer Prev, 13, 957-62.

Osoba D, Aaronson N, Zee B, et al (1997). Modification of the EORTC QLQ-C30 (version 2.0) based on content validity and reliability testing in large samples of patients with cancer. the study group on quality of life of the EORTC and the symptom control and quality of life committees of the NCI of canada clinical trials group. Qual Life Res, 6, 103-8.

Pachler J, Wille-Jorgensen P (2012). Quality of life after rectal resection for cancer, with or without permanent colostomy. Cochrane Database Syst Rev, 12, 4323.

Paiva CE, Carneseca EC, Barroso EM, et al (2014). Further evaluation of the EORTC QLQ-C30 psychometric properties in a large Brazilian cancer patient cohort as a function of their educational status. Support Care Cancer.

Perwitasari DA, Atthobari J, Dwiprahasto I, et al (2011). Translation and validation of EORTC QLQ-C30 into Indonesian version for cancer patients in Indonesia. Jpn J Clin Oncol, 41, 519-29.

Schwenk W, Neudecker J, Haase O, et al (2004). Comparison of EORTC quality of life core questionnaire (EORTCQLQ-C30) and gastrointestinal quality of life index (GIQLI) in patients undergoing elective colorectal cancer resection. Int J Colorectal Dis, 19, 554-60.

Shah SA, Neoh HM, Rahim SS, et al (2014). Spatial analysis of colorectal cancer cases in Kuala Lumpur. Asian Pac J Cancer Prev, 15, 1149-54.

Sharifa Ezat WP, Fuad I, Hayati Y, et al (2014). Observational study on patient's satisfactions and quality of life (QoL) among cancer patients receiving treatment with palliative care intent in a tertiary hospital in Malaysia. Asian Pac J Cancer Prev, 15, 695-701.

Smith AB, Cocks K, Parry D, et al (2013). Reporting of healthrelated quality of life (HRQOL) data in oncology trials: a comparison of the european organization for research and treatment of cancer quality of life (EORTC QLQ-C30) and the functional assessment of cancer therapy-general (FACT-G). Qual Life Res.

Uwer L, Rotonda C, Guillemin F, et al (2011). Responsiveness of EORTC QLQ-C30, QLQ-CR38 and FACT-C quality of life questionnaires in patients with colorectal cancer. Health Qual Life Outcomes, 9, 70.

Velikova G, Coens C, Efficace F, et al (2012). Health-Related Quality of Life in EORTC clinical trials - 30 years of progress from methodological developments to making a real impact on oncology practice. Eur J Cancer Supplements, 10, 141-9.

Wan C, Meng Q, Yang Z, et al (2008). Validation of the simplified Chinese version of EORTC QLQ-C30 from the measurements of five types of inpatients with cancer. Ann Oncol, 19, 2053-60.

Yakar HK, Pinar R (2013). Reliability and validity of Turkish version of the caregiver quality of life index cancer scale. Asian Pac J Cancer Prev, 14, 4415-9.
Yun YH, Park YS, Lee E, et al (2004). Validation of the Korean version of the EORTC QLQ-C30. Quality Life Res, 13, 863-8.

Yusoff N, Low WY, CH Y (2010). The Malay version of the European organization for research and treatment of cancer quality of life questionnaire (EORTC-QLQ C30): realibility and validity study. Int Med J Malaysia, 9, 45-50.

Zaj ACO, Spycha LAA, Murawa D, et al (2008). Quality of life assessment in patients with a stoma due to rectal cancer. Reports Practical Oncol Radiotherapy, 13, 130-4. 